\title{
Three-dimensional unsteady heat conduction analysis by the triple-reciprocity boundary element method
}

\author{
Y. Ochiai \& Y. Kitayama \\ Department of Mechanical Engineering, Kinki University, Japan
}

\begin{abstract}
The conventional boundary element method (BEM) requires a domain integral in heat conduction analysis with heat generation or an initial temperature distribution. In this paper it is shown that the three-dimensional heat conduction problem can be solved effectively using the triple-reciprocity boundary element method without internal cells. In this method, the distributions of heat generation and initial temperature are interpolated using integral equations and timedependent fundamental solutions are used. A new computer program was developed and applied to solving several problems.
\end{abstract}

Keywords: boundary element method, heat conduction, meshless method.

\section{Introduction}

The unsteady heat conduction problem without arbitrary heat generation and a nonuniform initial temperature distribution can be easily solved, without using internal cells, by the conventional boundary element method (BEM). For special cases, unsteady heat conduction problems with constant heat generation and uniform initial temperature distribution can solved by the standard BEM without the need for internal cells. When an analysis of heat conduction under arbitrary heat generation or a non-uniform initial temperature distribution within the domain is carried out by the BEM, a domain integral is generally necessary [1, 2]. However, by including the domain integral, the merit of BEM, that the preparation of data is simple, is lost. Thus, several other methods have been considered. Nowak and Neves proposed a multiple-reciprocity method [3]. Tanaka et al. have proposed a dual-reciprocity BEM for transient heat conduction problems, and V. Sladek and J. Sladek proposed a local boundary 
integral equation for unsteady heat conduction problems. However, these methods do not employ a time-dependent fundamental solution, which gives an accurate result.

Ochiai proposed the triple-reciprocity BEM or improved multi-reciprocity BEM for steady heat conduction, steady thermal stress and elastoplastic problems [4-6]. The triple-reciprocity BEM for two-dimensional heat conduction and thermal stress analysis for an unsteady state has also been proposed [7, 8]. In this paper the triple-reciprocity BEM is used for threedimensional unsteady heat conduction problems. In this method, heat generation and the initial temperature distributions are interpolated using the boundary integral equations. The triple-reciprocity method, which does not require internal cells, uses a time-dependent solution.

\section{Theory}

\subsection{Unsteady heat conduction}

In unsteady heat conduction problems with heat generation $W_{1}^{S}(q, t)$, a temperature $T$ is obtained by solving

$$
\nabla^{2} T+\frac{W_{1}^{S}}{\lambda}=\kappa^{-1} \frac{\partial T}{\partial t},
$$

where $\kappa, \lambda$ and $t$ are the thermal diffusivity, heat conductivity and time, respectively. Denoting an arbitrary time and the initial temperature by $\tau$ and $T^{0 S}(q, 0)$, respectively, the boundary integral equation for the temperature in the case of unsteady heat conduction problems is expressed by $[1,2]$

$$
\begin{aligned}
c T(P, t)= & -\kappa \int_{0}^{t} \int_{\Gamma}\left[T(Q, \tau) \frac{\partial T_{1}^{*}(P, Q, t, \tau)}{\partial n}-\frac{\partial T(Q, \tau)}{\partial n} T_{1}^{*}(P, Q, t, \tau)\right] d \Gamma d \tau \\
& +\kappa \int_{0}^{t} \int_{\Omega} T_{1}^{*}(P, q, t, \tau) \frac{W_{1}^{S}(q, \tau)}{\lambda} d \Omega d \tau+\int_{\Omega} T_{1}^{*}(P, q, t, 0) T_{1}^{0 S}(q, 0) d \Omega,
\end{aligned}
$$

where $c=0.5$ on the smooth boundary and $c=1$ in the domain. $\Gamma$ and $\Omega$ represent the boundary and the domain, respectively, $p$ and $q$ are respectively an observation point and a loading point, and $r$ is the distance between $p$ and $q$. The notations $p$ and $q$ are written as $P$ and $Q$ on the boundary, respectively. In the case of three-dimensional problems, the time-dependent fundamental solution $T_{1}^{*}(p, q, t, \tau)$ in Eq. (2) for the unsteady temperature analysis problem and its normal derivative are given by $[1,2]$

$$
\begin{aligned}
T_{1}^{*}(p, q, t, \tau) & =\frac{1}{[4 \pi \kappa(t-\tau)]^{3 / 2}} \exp [-a] \\
\frac{\partial T_{1}^{*}(p, q, t, \tau)}{\partial n} & =\frac{-2 r}{\pi^{3 / 2}[4 \kappa(t-\tau)]^{5 / 2}} \frac{\partial r}{\partial n} \exp (-a)
\end{aligned}
$$

where 


$$
a=\frac{r^{2}}{4 \kappa(t-\tau)}
$$

As shown in Eq. (2), when there is an arbitrary initial temperature or heat generation distribution, a domain integral becomes necessary.

\subsection{Interpolation}

An interpolation method for a distribution of heat generation $W_{1}^{S}(q, \tau)$ is shown using the boundary integral equations to avoid the use of internal cells. The polyharmonic function $T_{1}^{[f]}(p, q)$ for the steady state is given by

$$
T^{[f]}(P, Q)=\frac{r^{2 f-3}}{4 \pi(2 f-2) !} .
$$

In reference [15], the polyharmonic function of body distribution is used. However, the corresponding functions for the unsteady three-dimensional case are very difficult to obtain. In this paper, the polyharmonic functions of the surface distribution are used. Figure 1 shows the shape of polyharmonic functions; the biharmonic function $T^{[2]}$ is not smooth at $r=0$. In the threedimensional case, smooth interpolation cannot be achieved using only the biharmonic function $T^{[2]}$. To achieve smooth interpolation, the polyharmonic function with surface distribution $T^{[2] A}$ is introduced. A polyharmonic function with surface distribution $T^{[f] A}$, as shown in Fig. 2, is defined as [14]

$$
T^{[f] A}=\int_{0}^{2 \pi}\left(\int_{0}^{\pi} T^{[f]} A^{2} \sin \theta d \theta\right) d \phi .
$$

$T^{[f] A}$ can be easily obtained using the relationships $r^{2}=R^{2}+A^{2}-2 A R \cos \theta$ and $d r=A R \sin \theta d \theta$, as shown in Fig. 2. This function is written using $r$ instead of $R$, similarly to Eqs. (3) and (6), although the function in Eq. (7) is a function of $R$. The newly defined function $T^{[f] A}$ can be explicitly written as

$$
\begin{array}{ll}
T^{[f] A}=\frac{A\left\{(r+A)^{2 f-1}-(r-A)^{2 f-1}\right\}}{2(2 f-1) ! r} & r>A \\
T^{[f] A}=\frac{A\left\{(A+r)^{2 f-1}-(A-r)^{2 f-1}\right\}}{2(2 f-1) ! r} & r \leq A .
\end{array}
$$
[9]:

The following equations can be used for the three-dimensional interpolation

$$
\begin{gathered}
\nabla^{2} W_{1}^{S}(q, \tau)=-W_{2}^{S}(q, \tau) \\
\nabla^{2} W_{2}^{S}(q, \tau)=-\sum_{m=1}^{M} W_{3}^{P A}\left(q_{m}, \tau\right) \delta\left(q-q_{m}\right)
\end{gathered}
$$

$M$ is the number of internal points for interpolation. Assuming the spatial distribution of $W_{2}^{s}(q, \tau)$ to be governed by Eq. (11) with point sources, it is known that $W_{2}^{s}(q, \tau)$ will be divergent at these source points as the particular 
solution $\sum_{m=1}^{M} T^{[1]}\left(p, q_{m}\right) W_{3}^{P A}\left(q_{m}, \tau\right)$. Nevertheless, we can evaluate $W_{2}^{S}(q, \tau)$ on the boundary. The term $W_{2}^{S}$ of Eq. (10) corresponds to the sum of the curvatures $\partial^{2} W_{1}^{S} / \partial x^{2}, \partial^{2} W_{1}^{S} / \partial y^{2}$ and $\partial^{2} W_{1}^{S} / \partial z^{2}$. The term $W_{3}^{P A}$ is the unknown strength of a Dirac function. From Eqs. (10) and (11), the following equation can be obtained.

$$
\nabla^{4} W_{1}^{S}(q, \tau)=\sum_{m=1}^{M} W_{3}^{P A}\left(q_{m}, \tau\right) \delta\left(q-q_{m}\right)
$$

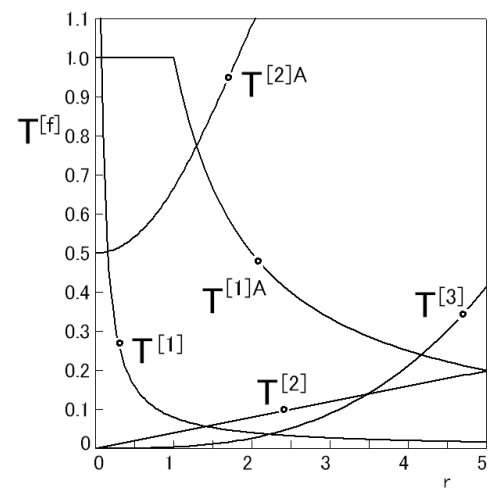

Figure 1: Polyharmonic functions.

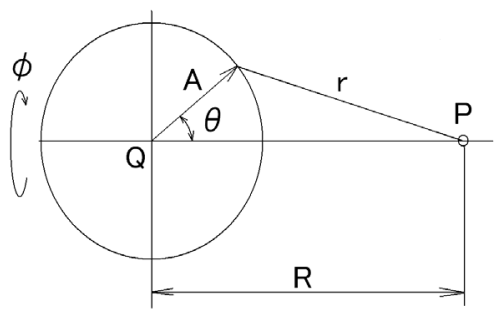

Figure 2: Notation for polyharmonic function with surface distribution.

This equation corresponds to equation for the deformation of an idealized thin plate with $\mathrm{M}$ point loads. The deformation $W_{1}^{S}(q, \tau)$ is given, but the force of the point load $W_{3}^{P A}(q, \tau)$ is unknown. $W_{3}^{P A}(q, \tau)$ is obtained inversely from the deformation $W_{1}^{S}(q, \tau)$ of the fictitious thin plate. $W_{2}^{S}$ corresponds to the moment of the thin plate. The moment $W_{2}^{S}$ on the boundary is assumed to be 0 , which is the same as that in a natural spline. This indicates that the thin plate is simply supported. Moreover, the distribution of the initial temperature can be interpolated as follows.

$$
\begin{gathered}
\nabla^{2} T_{1}^{0 S}(q, 0)=-T_{2}^{0 S}(q, 0) \\
\nabla^{2} T_{2}^{0 S}(q, 0)=-\sum_{m=1}^{M} T_{3}^{0 P A}\left(q_{m}, 0\right) \delta\left(q-q_{m}\right)
\end{gathered}
$$

On the other hand, the polyharmonic function $T_{f}^{*}(p, q, t, \tau)$ in the unsteady heat conduction problem and $T_{f A}^{*}(p, q, t, \tau)$ are defined by

$$
\nabla^{2} T_{f+1}^{*}(p, q, t, \tau)=T_{f}^{*}(p, q, t, \tau)
$$




$$
T_{f}^{* A}(p, q, t, \tau)=\int_{0}^{2 \pi}\left[\int_{0}^{\pi} T_{f}^{*}(p, q, t, \tau) A^{2} \sin \theta d \theta\right] d \phi
$$

Using Green's theorem twice, and Eqs. (10)-(16), Eq. (2) becomes

$$
\begin{aligned}
& c T(P, t)=-\kappa \int_{0}^{t} \int_{\Gamma}\left[T(Q, \tau) \frac{\partial T_{1}^{*}(P, Q, t, \tau)}{\partial n}-\frac{\partial T(Q, \tau)}{\partial n} T_{1}^{*}(P, Q, t, \tau)\right] d \Gamma d \tau \\
& +\frac{\kappa}{\lambda} \sum_{f=1}^{2}(-1)^{f} \int_{0}^{t} \int_{\Gamma}\left[T_{f+1}^{*}(P, Q, t, \tau) \frac{\partial W_{f}^{S}(Q, \tau)}{\partial n}\right. \\
& \left.-\frac{\partial T_{f+1}^{*}(P, Q, t, \tau)}{\partial n} W_{f}^{S}(Q, \tau)\right] d \Gamma d \tau+\frac{\kappa}{\lambda} \sum_{m=1}^{M} \int_{0}^{t} W_{3(m)}^{P}(q, \tau) T_{3}^{* A}(P, q, t, \tau) d \tau \\
& +\sum_{f=1}^{2}(-1)^{f} \int_{\Gamma}\left[T_{f+1}^{*}(P, Q, t, 0) \frac{\partial T_{f}^{0 S}(Q, 0)}{\partial n}-\frac{\partial T_{f+1}^{*}(P, Q, t, 0)}{\partial n} T_{f}^{0 S}(Q, 0)\right] d \Gamma \\
& +\sum_{m=1}^{M} T_{3}^{0 P A}\left(q_{m}, 0\right) T_{3}^{* A}\left(P, q_{m}, t, 0\right) .
\end{aligned}
$$

Using Green's second identity and Eqs. (10) and (11), we obtain for $W_{1}^{S}$ and $W_{2}^{S}[7-9]$

$$
\begin{aligned}
c W_{1}^{S}(P, \tau)= & \sum_{f=1}^{2}(-1)^{f} \int_{\Gamma}\left\{T^{[f]}(P, Q) \frac{\partial W_{f}^{S}(Q, \tau)}{\partial n}\right. \\
& \left.-\frac{\partial T^{[f]}(P, Q)}{\partial n} W_{f}^{S}(Q, \tau)\right\} d \Gamma-\sum_{m=1}^{M} T^{[2] A}(P, q) W_{3}^{P A}\left(q_{m}, \tau\right) \\
c W_{2}^{S}(P, \tau)= & \int_{\Gamma}\left\{T^{[1]}(P, Q) \frac{\partial W_{2}^{S}(Q, \tau)}{\partial n}\right. \\
& \left.-\frac{\partial T^{[1]}(P, Q)}{\partial n} W_{2}^{S}(Q, \tau)\right\} d \Gamma+\sum_{m=1}^{M} T^{[1] A}\left(P, q_{m}\right) W_{3}^{P A}\left(q_{m}, \tau\right)
\end{aligned}
$$

\subsection{Unsteady polyharmonic function}

The three-dimensional unsteady polyharmonic function $T_{f}^{*}(P, q, t, \tau)$ in Eq. (17) is determined as

$$
T_{f+1}^{*}(p, q, t, \tau)=\int \frac{1}{r^{2}} \int r^{2} T_{f}^{*}(p, q, t, \tau) d r d r
$$

The polyharmonic function $T_{f}^{*}(P, q, t, \tau)$ in the unsteady state and its normal derivative are explicitly given by

$$
\begin{gathered}
T_{2}^{*}(q, p, t, \tau)=\frac{1}{2 \pi^{3 / 2} r}\left\{-\gamma(1.5, a)+a^{1 / 2}[1-\exp (-a)]\right\}=\frac{-1}{2 \pi^{3 / 2} r} \gamma(0.5, a) \\
\frac{\partial T_{2}^{*}(q, p, t, \tau)}{\partial n}=\frac{1}{2 \pi^{3 / 2} r^{2}} \gamma(1.5, a) \frac{\partial r}{\partial n}
\end{gathered}
$$




$$
\begin{aligned}
& T_{3}^{*}= \frac{r}{12 \pi^{3 / 2}}\left\{-3 \gamma(1.5, a)+6 a^{-1 / 2} \gamma(2, a)-3 a^{-1} \gamma(2.5, a)+a^{1 / 2}\right. \\
&\left.+3 \gamma(1.5, a) \frac{1}{a}-3 a^{-1 / 2}[1-\exp (-a)]\right\} \\
&= \frac{r}{4 \pi^{3 / 2}}\left\{-\gamma(0.5, a)+2 \gamma(1.5, a) \frac{1}{a}-2 a^{-1 / 2}[1-\exp (-a)]\right\} \\
& \frac{\partial T_{3}^{*}}{\partial n}=\frac{1}{4 \pi^{3 / 2}}\left[\gamma(0.5, a)-\frac{1}{a} \gamma(1.5, a)\right] \frac{\partial r}{\partial n},
\end{aligned}
$$

where $\gamma($,$) is an incomplete gamma function of the first kind and$ $r,{ }_{i}=\partial r / \partial x_{i}$. Using Eqs. (7) and (17), the polyharmonic function with a surface distribution is obtained as follows:

$$
\begin{aligned}
T_{3}^{* A}= & \frac{2 A(k t)^{3 / 2}}{3 \pi^{1 / 2} r}\left\{-2 u_{2}{ }^{3 / 2} \gamma\left(1.5, u_{2}\right)+2 u_{1}^{3 / 2} \gamma\left(1.5, u_{1}\right)+2 \gamma\left(3, u_{2}\right)\right. \\
& -2 \gamma\left(3, u_{1}\right)+6 u_{2} \gamma\left(2, u_{2}\right)-6 u_{1} \gamma\left(2, u_{1}\right)-6 u_{2}{ }^{1 / 2} \gamma\left(2.5, u_{2}\right)+6 u_{1}{ }^{1 / 2} \gamma\left(2.5, u_{1}\right) \\
& +\frac{1}{2} u_{2}{ }^{2}-\frac{1}{2} u_{1}{ }^{2}+6 u_{2}{ }^{1 / 2} \gamma\left(1.5, u_{2}\right)-6 u_{1}{ }^{1 / 2} \gamma\left(1.5, u_{1}\right)-6 \gamma\left(2, u_{2}\right) \\
& \left.+6 \gamma\left(2, u_{1}\right)-3 u_{2}+3 u_{1}-3 \exp \left(-u_{2}\right)+3 \exp \left(-u_{1}\right)\right\}
\end{aligned}
$$

where

$$
u_{1}=\frac{(r-A)^{2}}{4 \kappa(t-\tau)} \quad, \quad u_{2}=\frac{(r+A)^{2}}{4 \kappa(t-\tau)}
$$

Numerical solutions are obtained using the interpolation functions for time and space. If a constant time interpolation and time step $\left(t_{k}-t_{k-1}\right)$ are used, the time integral can be treated analytically. The time integrals for $T_{f}^{*}(P, q, t, \tau)$ and $\partial T_{f}^{*} / \partial n$ from $t_{f}$ to $t_{F}$ are given as follows:

$$
\begin{gathered}
\int_{t_{f}}^{t_{F}} T_{1}^{*}(p, q, t, \tau) d \tau=\frac{1}{4 \kappa \pi^{3 / 2} r} \Gamma\left(0.5, a_{f}\right) \\
\int_{t_{f}}^{t_{F}} \frac{\partial T_{1}^{*}(p, q, t, \tau)}{\partial n} d \tau=\frac{1}{2 \kappa \pi^{3 / 2} r^{2}} \frac{\partial r}{\partial n} \Gamma\left(1.5, a_{f}\right) \\
\int_{t_{f}}^{t_{F}} T_{2}^{*}(p, q, t, \tau) d \tau=\frac{r}{8 \kappa \pi^{3 / 2}}\left[\gamma\left(1.5, a_{f}\right) \frac{-1}{a_{f}}-\Gamma\left(0.5, a_{f}\right)+\frac{2}{a_{f}{ }^{1 / 2}}-\Gamma\left(-0.5, a_{f}\right)\right] \\
=\frac{r}{8 \kappa \pi^{3 / 2}}\left[\gamma\left(0.5, a_{f}\right) \frac{1}{a_{f}}-\Gamma\left(-0.5, a_{f}\right)\right] \\
\int_{t_{f}}^{t_{F}} \frac{\partial T_{2}^{*}(p, q, t, \tau)}{\partial n} d \tau=\frac{1}{8 \kappa \pi^{3 / 2}} \frac{\partial r}{\partial n}\left[\gamma\left(1.5, a_{f}\right) \frac{1}{a_{f}}+\Gamma\left(0.5, a_{f}\right)\right]
\end{gathered}
$$




$$
\begin{aligned}
& \int_{t_{f}}^{t_{F}} T_{3}^{*}(p, q, t, \tau) d \tau=\frac{r^{3}}{96 \kappa \pi^{3 / 2}}\left[-6 \gamma\left(1.5, a_{f}\right) \frac{1}{a_{f}}-\Gamma\left(0.5, a_{f}\right)+8 \gamma\left(2, a_{f}\right) \frac{1}{a_{f}^{3 / 2}}\right. \\
& -3 \gamma\left(2.5, a_{f}\right) \frac{1}{a_{f}^{2}}+\frac{4}{a_{f}^{1 / 2}}+3 \gamma\left(1.5, a_{f}\right) \frac{1}{a_{f}^{2}}+3 \Gamma\left(-0.5, a_{f}\right) \\
& \left.-\frac{4}{a_{f}^{3 / 2}}+6 \Gamma\left(-1.5, a_{f}\right)\right] \\
& \int_{t_{f}}^{t_{F}} \frac{\partial T_{3}^{*}(p, q, t, \tau)}{\partial n} d \tau=\frac{r^{2}}{96 \kappa \pi^{3 / 2}} \frac{\partial r}{\partial n}\left[-6 \gamma\left(1.5, a_{f}\right) \frac{1}{a_{f}}-3 \Gamma\left(0.5, a_{f}\right)\right. \\
& \left.\quad+3 \gamma\left(2.5, a_{f}\right) \frac{1}{a_{f}{ }^{2}}+\frac{8}{a_{f}^{1 / 2}}-\gamma\left(1.5, a_{f}\right) \frac{3}{a_{f}^{2}}-3 \Gamma\left(-0.5, a_{f}\right)\right]
\end{aligned}
$$

where

$$
a_{f}=\frac{r^{2}}{4 \kappa\left(t_{F}-t_{f}\right)}
$$

$\Gamma($,$) is an incomplete gamma function of the second kind. The time integral$ of Eq. (25) can be obtained as follows:

$$
\begin{aligned}
& \int_{t_{f}}^{t_{F}} T_{3}^{* A}(p, q, t, \tau) d \tau=\frac{A}{48 \pi^{1 / 2} r} \frac{(r-A)^{5}}{\kappa}\left\{2 \gamma\left(1.5, a_{1 f}\right) \frac{1}{a_{f}}+\frac{1}{5} \Gamma\left(0.5, a_{1 f}\right)\right. \\
& -\frac{4}{5} \gamma\left(3, a_{1 f}\right) \frac{1}{a_{1 f}^{5 / 2}}-4 \gamma\left(2, a_{1 f}\right) \frac{1}{a_{1 f}^{3 / 2}}+3 \gamma\left(2.5, a_{1 f}\right) \frac{1}{a_{1 f}{ }^{2}}-\frac{1}{a_{1 f}{ }^{1 / 2}} \\
& -3 \gamma\left(1.5, a_{1 f}\right) \frac{1}{a_{1 f}{ }^{2}}-\frac{3}{5} \Gamma\left(-0.5, a_{1 f}\right) \\
& \left.+\frac{12}{5} \gamma\left(2, a_{1 f}\right) \frac{1}{a_{1 f}^{5 / 2}}+\frac{2}{a_{1 f}^{3 / 2}}+3 \Gamma\left(-2.5, a_{1 f}\right)\right\},
\end{aligned}
$$

where

$$
a_{1 f}=\frac{(r-A)^{2}}{4 \kappa\left(t_{F}-t_{f}\right)} .
$$

For the sake of conciseness, the terms involving $u_{2}$ in Eq. (34) are omitted.

If there are no arbitrary heat generation or initial temperature distributions, internal points are not necessary. Also, for the special case of the formulation of an unsteady state from a steady state, internal points are not necessary. If the heat generation and initial temperature distributions are governed by Laplace equation instead of Eqs. (10)-(14), Eq. (17) becomes

$$
\begin{aligned}
& c T(P, t)=-\kappa \int_{0}^{t} \int_{\Gamma}\left[T(Q, \tau) \frac{\partial T_{1}^{*}(P, Q, t, \tau)}{\partial n}-\frac{\partial T(Q, \tau)}{\partial n} T_{1}^{*}(P, Q, t, \tau)\right] d \Gamma d \tau \\
& +\frac{\kappa}{\lambda} \int_{0}^{t} \int_{\Gamma}\left[T_{2}^{*}(P, Q, t, \tau) \frac{\partial W_{1}^{S}(Q, \tau)}{\partial n}-\frac{\partial T_{2}^{*}(P, Q, t, \tau)}{\partial n} W_{1}^{S}(Q, \tau)\right] d \Gamma d \tau
\end{aligned}
$$




$$
+\int_{\Gamma}\left[T_{2}^{*}(P, Q, t, 0) \frac{\partial T_{1}^{0 S}(Q, 0)}{\partial n}-\frac{\partial T_{2}^{*}(P, Q, t, 0)}{\partial n} T_{1}^{0 S}(Q, 0)\right] d \Gamma
$$

\section{Numerical examples}

To verify the efficiency of this method, an unsteady temperature distribution in a sphere is obtained. The initial temperature of the sphere is $T_{0}=10^{\circ} \mathrm{C}$, and the temperature on the surface suddenly becomes $0^{\circ}$ at time $t=0$. It is assumed that the thermal diffusivity is $\kappa=16 \mathrm{~mm}^{2} \mathrm{~s}^{-1}$ and the radius of the sphere is $b=10 \mathrm{~mm}$. Figure 3 shows the boundary elements. In this example, Eq. (36) is used; therefore, internal points are not necessary. Figure 4 shows the temperature change. The solid lines in Fig.4 show the exact solutions, which are given by

$$
T(r, t)=\frac{2 b T_{0}}{\pi r} \sum_{n=1}^{\infty} \frac{(-1)^{n+1}}{n} \sin \frac{n \pi r}{b} \exp \left(-\frac{\kappa n^{2} \pi^{2} t}{b^{2}}\right) .
$$

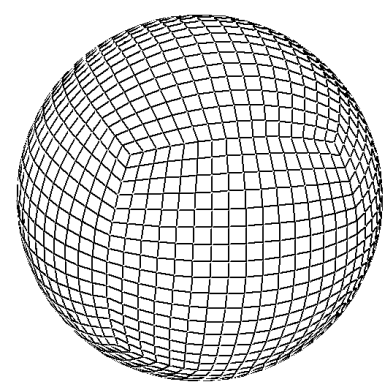

Figure 3: Boundary elements of spherical region.

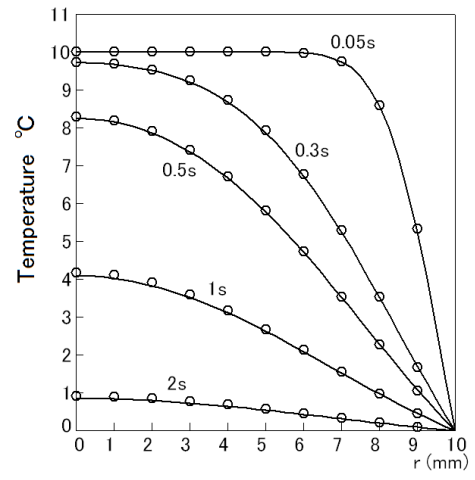

Figure 4: Temperature distributions in sphere.

The next numerical example is a cubic region with length $L=10 \mathrm{~mm}$ with heat generation. Using internal points as shown in Fig. 5, it is assumed that the thermal diffusivity $\kappa$ is $16 \mathrm{~mm}^{2} \mathrm{~s}^{-1}$. The number of boundary elements and internal points are 600 and $M=729$, respectively. The surface temperature is $0^{\circ} \mathrm{C}$ and the initial temperature is $0^{\circ} \mathrm{C}$. Step heating is assumed. The heat generation is given by

$$
W(x, y, z)=W_{0} \sin \frac{\pi x}{L} \sin \frac{\pi y}{L} \sin \frac{\pi z}{L} \quad(t \geq 0) .
$$

Using a Laplace transformation and a finite sine transformation, an exact solution is obtained as follows:

$$
T(x, y, z, t)=\frac{\kappa W_{0}}{\lambda E} \sin \frac{\pi x}{L} \sin \frac{\pi y}{L} \sin \frac{\pi z}{L}[1-\exp (-E t)]
$$




$$
E=\frac{3 \kappa \pi^{2}}{L^{2}}
$$

$W_{0} / \lambda=10 \mathrm{~K} / \mathrm{mm}^{2}$ is assumed. Figure 6 shows the comparison between this method and exact solution given by Eq. (39) at $t=0.05,0.1,0.2,0.4$ and $1 \mathrm{~s}$.

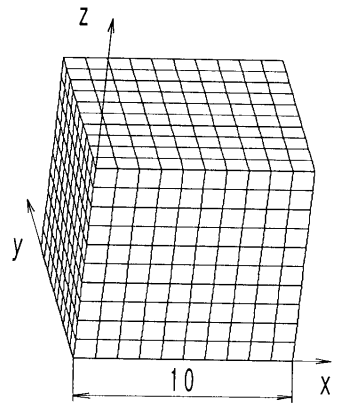

(a) Boundary elements

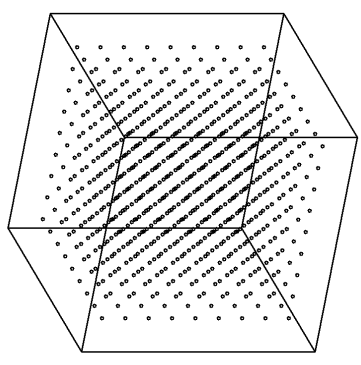

(b) Internal points

Figure 5: Cubic region.

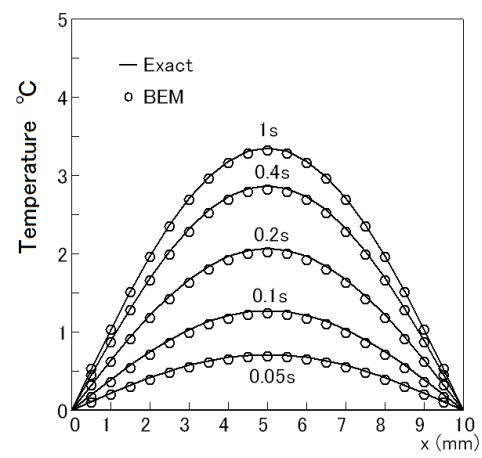

Figure 6: Temperature distributions in cube $(y=z=5)$.

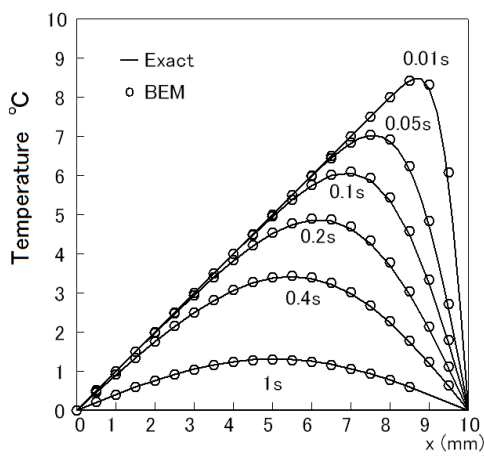

Figure 7: Temperature distributions in cube $(y=z=5)$.

For the special case of the formulation of an unsteady state from a steady state, internal points are not necessary. Temperature of cubic region is obtained using boundary elements as shown in Fig. 5. In this calculation, internal points are not necessary. The thermal diffusivity $\kappa$ is $16 \mathrm{~mm}^{2} \mathrm{~s}^{-1}$. The temperatures at $x=0$ and $x=10$ are $0^{\circ} \mathrm{C}$ and $T_{0}=10^{\circ} \mathrm{C}$, respectively. The other surfaces are adiabatic. Initial temperature is given by

$$
T(x, 0)=\frac{T_{0} x}{L} .
$$


The temperature at $x=10$ suddenly becomes $T_{0}=0{ }^{\circ} \mathrm{C}$ at time $t=0 \mathrm{~s}$. The unsteady temperature distribution is obtained by Eq. (36). Using a Laplace transformation and a finite sine transformation, the exact solution is obtained as follows:

$$
T(x, t)=\frac{-2 T_{0}}{\pi} \sum_{n=1}^{\infty} \frac{\cos (\pi n)}{n} \sin \frac{\pi n x}{L} \exp \left(-\frac{\kappa n^{2} \pi^{2} t}{L^{2}}\right) .
$$

Figure 7 shows the comparison between this method and the exact solution given by Eq. (42) at $t=0.01,0.05,0.1,0.2,0.4$ and $1 \mathrm{~s}$.

\section{Conclusion}

It has been shown that it is possible to express the distributions of heat generation and initial temperature for the three-dimensional case using only the fundamental solution of lower order by the triple-reciprocity boundary element method. It has also been shown that highly accurate unsteady heat conduction analysis using the boundary integral is only possible using the polyharmonic function and the surface-distributed polyharmonic function, even in the case of arbitrary distributions of heat generation and initial temperature. Therefore, by adding only the data of the values at internal points and on the boundary for the distributions of heat generation and initial temperature, the analysis of threedimensional heat conduction for the unsteady state with heat generation and initial temperature distributions has become possible. A reduction of the dimensionality of the problem has been effectively achieved.

\section{References}

[1] C. A. Brebbia, J. C. F. Telles and L. C. Wrobel, Boundary Element Techniques - Theory and Applications in Engineering, pp. 47-107, Berlin, Springer-Verlag, 1984.

[2] L. C. Wrobel, The Boundary Element Method, Volume 1, John Wiley \& Sons, West Sussex, pp.97-117 (2002).

[3] Nowak, A. J., and Neves, A. C., The Multiple Reciprocity Boundary Element Method, Computational Mechanics Publication, Southampton, Boston, (1994).

[4] Ochiai, Y. and Sekiya, T., Steady Heat Conduction Analysis by Improved Multiple-Reciprocity Boundary Element Method, Engineering Analysis with Boundary Elements, Vol. 18, pp. 111-117, (1996).

[5] Ochiai, Y. and Kobayashi, T., Initial Strain Formulation without Internal Cells for Elastoplastic Analysis by Triple-Reciprocity BEM, International Journal for Numerical Methods in Engineering, Vol. 50, pp. 1877-1892, (2001).

[6] Y. Ochiai, Two-Dimensional Unsteady Heat Conduction Analysis with Heat Generation by Triple-Reciprocity BEM, International Journal of Numerical Methods in Engineering, Vol. 51, No. 2, pp. 143-157(2001). 
[7] Y. Ochiai, V. Sladek and J. Sladek, Transient Heat Conduction Analysis by Triple-Reciprocity Boundary Element Method, Engineering Analysis with Boundary Elements, Vol. 30, pp. 194-204 (2006).

[8] Ochiai, Y., Two-Dimensional Unsteady Thermal Stress Analysis by TripleReciprocity Boundary Element Method, Journal of Thermal Stresses, Vol. 24, No. 3, pp. 233-253, (2001).

[9] Y. Ochiai and V. Sladek, Numerical Treatment of Domain Integrals without Internal Cells in Three-Dimensional BIEM Formulations, CMES (Computer Modeling in Engineering \& Sciences), Vol. 6, No. 6, pp. 525-536, 2004. 